\title{
CONVERGENCE OF EXTENDED LAGRANGE INTERPOLATION
}

\author{
GIULIANA CRISCUOLO, GIUSEPPE MASTROIANNI, AND DONATELLA OCCORSIO
}

\begin{abstract}
The authors give a procedure to construct extended interpolation formulae and prove some uniform convergence theorems.
\end{abstract}

\section{INTRODUCTION}

Let $X=\left\{x_{m, i}, i=1, \ldots, m, m \in N\right\}$ be a matrix of knots belonging to $I:=[-1,1]$. For a given bounded function $f$, the corresponding Lagrange polynomial interpolating at the points $x_{m, i}, i=1, \ldots, m$, is denoted by $L_{m}(X ; f)$. If $Y=\left\{y_{n, j}, j=1, \ldots, n, n \in N\right\}$ is another matrix of knots belonging to $I$, then we define the "extended interpolation polynomial" as the Lagrange polynomial $L_{m+n}(X, Y ; f)$ of degree $m+n-1$ which interpolates the function $f$ at the points $x_{m, i}, i=1, \ldots, m$, and $y_{n, j}, j=1, \ldots, n$. Since

$$
L_{m+n}(X, Y ; f)=q_{n} L_{m}\left(X ; f q_{n}^{-1}\right)+p_{m} L_{n}\left(Y ; f p_{m}^{-1}\right)
$$

where

$$
p_{m}(x)=\prod_{i=1}^{m}\left(x-x_{m, i}\right), \quad q_{n}(x)=\prod_{j=1}^{n}\left(x-y_{n, j}\right),
$$

the extended interpolating polynomial makes sense when the polynomials $p_{m}$ and $q_{n}$ have no common zeros for every fixed $m, n \in N$.

Even though it is easy to construct extended interpolating formulae, the study of their convergence is difficult, in general. Extended interpolation processes have been proposed to find the numerical solution of functional equations [ 1 , 2], and they are used especially for numerical quadrature (extended quadrature formulae). Quadratures of this type have been studied by several authors (see $[5,6,9])$.

The main purpose of this paper is to give a new method of constructing "good" formulae of extended interpolation $L_{m+n}(X, Y ; f)$; namely, we shall assume that the elements of the matrix $X$ coincide with the zeros of some orthogonal polynomials in $I$ with respect to a weight function $w$ and then construct the knots of the matrix $Y$ so that they are also zeros of orthogonal

Received March 24, 1989; revised July 28, 1989.

1980 Mathematics Subject Classification (1985 Revision). Primary 41A05. 
polynomials in $I$ with respect to a weight related to $w$. For each extended interpolation formulae we shall prove uniform convergence theorems.

\section{EXTENDED INTERPOLATION FORMULAE}

Let $d \mu$ be a finite positive measure on $[-1,1]$ with an infinite set as support and let $p_{m}(d \mu ; x)=\gamma_{m}(d \mu) x^{m}+\cdots, \gamma_{m}(d \mu)>0, m \in N$, be the corresponding system of orthonormal polynomials, that is

$$
\int_{-1}^{1} p_{m}(d \mu ; x) p_{n}(d \mu ; x) d \mu(x)=\delta_{m, n} .
$$

We write the three-term recurrence formula satisfied by $p_{m}(d \mu)$ as

$$
\begin{gathered}
x p_{m}(d \mu ; x)=a_{m+1} p_{m+1}(d \mu ; x)+b_{m} p_{m}(d \mu ; x)+a_{m} p_{m-1}(d \mu ; x) \\
p_{-1}(d \mu ; x)=0
\end{gathered}
$$

where

$$
\begin{gathered}
m=0,1, \ldots ; \quad a_{m}=a_{m}(d \mu)=\gamma_{m-1}(d \mu) / \gamma_{m}(d \mu) \\
b_{m}=b_{m}(d \mu)=\int_{-1}^{1} x p_{m}\{d \mu ; x)^{2} d \mu(x) .
\end{gathered}
$$

We now say that the measure $d \mu$ is in the class $M$ (Nevai's class) if

$$
\lim _{m \rightarrow \infty} a_{m}(d \mu)=\frac{1}{2} \text { and } \lim _{m \rightarrow \infty} b_{m}(d \mu)=0 .
$$

The class $M$ is sufficiently large to be of significant interest and has been thoroughly studied in [11]. For our purpose it is enough to know that if $d \mu(x)=w(x) d x, \operatorname{supp}(d \mu)=[-1,1]$, and $w(x)>0$ a.e. in $[-1,1]$, then $d \mu \in M[12$, p. 52].

We use in the sequel the following theorem which is a direct consequence of a result by Maté, Nevai, and Totik [8, p. 70, Theorem 11].

Theorem 2.1. Let $d \mu \in M$ and let $g$ be a nonnegative $d \mu$-integrable function. Assume, further, that there exists a polynomial $R$ such that $R g$ and $R g^{-1}$ are Riemann integrable in $[-1,1]$. Then $g d \mu \in M$ and

$$
\lim _{m \rightarrow \infty} \gamma_{m}(g d \mu) / \gamma_{m}(d \mu)=\exp \left\{-\frac{1}{2 \pi} \int_{-1}^{1} \frac{\log g(x)}{\sqrt{1-x^{2}}} d x\right\} .
$$

In the following we consider only $d \mu$-absolutely continuous measures. Then, letting $\left\{p_{m}(w)\right\}$ be the system of orthonormal polynomials corresponding to the weight function $w$, we denote by $X(w)$ the triangular matrix

$$
X(w)=\left\{x_{m, i}(w), i=1, \ldots, m, m \in N\right\},
$$

where $x_{m, i}(w), i=1, \ldots, m$, are the zeros of $p_{m}(w)$ ordered increasingly,

$$
-1<x_{m, 1}(w)<\cdots<x_{m, m}(w)<1 \text {. }
$$


The Christoffel constants $\lambda_{m, i}(w), i=1, \ldots, m$, are defined by $\lambda_{m, i}(w)=$ $\lambda_{m}\left(w ; x_{m, i}(w)\right)$, where

$$
\lambda_{m}(w ; x)=\left[\sum_{i=0}^{m-1} p_{i}^{2}(w ; x)\right]^{-1}
$$

is the $m$ th Christoffel function.

We are now able to state the following theorems which are essential for the construction of our interpolation formulae.

Theorem 2.2. If $w$ is any weight function and $\bar{w}(x)=\left(1-x^{2}\right) w(x)$, then the polynomial $Q_{2 m+1}=p_{m}(\bar{w}) p_{m+1}(w)$ satisfies

$$
\begin{gathered}
Q_{2 m+1}^{\prime}\left(x_{m, i}(\bar{w})\right)=-C_{m} \lambda_{m, i}^{-1}(\bar{w}), \quad i=1, \ldots, m, m \in N, \\
Q_{2 m+1}^{\prime}\left(x_{m+1, i}(w)\right)=C_{m} \lambda_{m+1, i}^{-1}(w)\left(1-x_{m+1, i}^{2}(w)\right)^{-1}, \\
i=1, \ldots, m+1, m \in N,
\end{gathered}
$$

where $C_{m}=\gamma_{m}(\bar{w}) \gamma_{m+1}^{-1}(w)+\gamma_{m+1}(w) \gamma_{m}^{-1}(\bar{w})<\infty$.

Hence, the zeros $x_{m, i}(\bar{w})$ of $p_{m}(\bar{w})$ interlace with the zeros $x_{m+1, i}(w)$ of $p_{m+1}(w)$, i.e.,

$$
x_{m+1, i}(w)<x_{m, i}(\bar{w})<x_{m+1, i+1}(w), \quad i=1, \ldots, m .
$$

Proof. We make use of a technique already used by Nevai in [10]. First, we consider the Fourier expansion of $\left(1-x^{2}\right) p_{m}(\bar{w} ; x)$ in the system $\left\{p_{k}(w)\right\}$ :

$$
\left(1-x^{2}\right) p_{m}(\bar{w} ; x)=\sum_{k=m}^{m+2} \bar{a}_{k} p_{k}(w ; x),
$$

where

$$
\bar{a}_{k}=\int_{-1}^{1} p_{k}(w ; x)\left(1-x^{2}\right) p_{m}(\bar{w} ; x) w(x) d x, \quad k=m, m+1, m+2 .
$$

In particular, $\bar{a}_{m}=\gamma_{m}(w) / \gamma_{m}(\bar{w})$ and $\bar{a}_{m+2}=-\gamma_{m}(\bar{w}) / \gamma_{m+2}(w)$ (cf. [10, p. 40]). Since

$$
x p_{m}(w ; x)=a_{m+1} p_{m+1}(w ; x)+b_{m} p_{m}(w ; x)+a_{m} p_{m-1}(w ; x)
$$

with $a_{m}=\gamma_{m-1}(w) / \gamma_{m}(w)$, we deduce

$$
p_{m+1}\left(w ; x_{m, i}(w)\right)=-\frac{\gamma_{m+1}(w) \gamma_{m-1}(w)}{\gamma_{m}^{2}(w)} p_{m-1}\left(w ; x_{m, i}(w)\right)
$$

This last relation, together with $(2.3)$, gives us

$$
\left(1-x_{m+1, i}^{2}(w)\right) p_{m}\left(\bar{w} ; x_{m+1, i}(w)\right)=A_{m} p_{m}\left(w ; x_{m+1, i}(w)\right)
$$

with

$$
A_{m}=\frac{\gamma_{m}(w)}{\gamma_{m}(\bar{w})}+\frac{\gamma_{m}(\bar{w}) \gamma_{m}(w)}{\gamma_{m+1}^{2}(w)}>0
$$


We now also consider the Fourier expansion of $p_{m+1}(w)$ in the system $\left\{p_{k}(\bar{w})\right\}$,

$$
p_{m+1}(w ; x)=\sum_{k=m-1}^{m+1} \bar{b}_{k} p_{k}(\bar{w} ; x)
$$

where

$$
\bar{b}_{k}=\int_{-1}^{1} p_{k}(\bar{w} ; x) p_{m+1}(w ; x)\left(1-x^{2}\right) w(x) d x, \quad k=m-1, m, m+1 .
$$

In particular, $\bar{b}_{m-1}=-\gamma_{m-1}(\bar{w}) / \gamma_{m+1}(w)$ and $\bar{b}_{m+1}=\gamma_{m+1}(w) / \gamma_{m+1}(\bar{w})$. Then by $(2.5)$,

$$
p_{m+1}\left(w ; x_{m, i}(\bar{w})\right)=-B_{m} p_{m-1}\left(\bar{w} ; x_{m, i}(\bar{w})\right)
$$

with

$$
B_{m}=\frac{\gamma_{m-1}(\bar{w})}{\gamma_{m+1}(w)}+\frac{\gamma_{m+1}(w) \gamma_{m-1}(\bar{w})}{\gamma_{m}^{2}(\bar{w})}>0
$$

Moreover, recalling Theorem 2.1, we conclude that the sequences $\left\{A_{m}\right\}$ and $\left\{B_{m}\right\}$ are convergent. In particular, the relations (2.4) and (2.6) give us that the zeros of $p_{m}(\bar{w})$ are different from the zeros of $p_{m+1}(w)$.

Now, since

$$
\begin{gathered}
Q_{2 m+1}^{\prime}\left(x_{m, i}(\bar{w})\right)=p_{m}^{\prime}\left(\bar{w} ; x_{m, i}(\bar{w})\right) p_{m+1}\left(w ; x_{m, i}(\bar{w})\right), \\
Q_{2 m+1}^{\prime}\left(x_{m+1, i}(w)\right)=p_{m+1}^{\prime}\left(w ; x_{m+1, i}(w)\right) p_{m}\left(\bar{w} ; x_{m+1, i}(w)\right),
\end{gathered}
$$

and recalling that for any weight $u$

$$
p_{m}^{\prime}\left(u ; x_{m, i}(u)\right)=\frac{\gamma_{m}(u)}{\gamma_{m-1}(u)} \frac{1}{p_{m-1}\left(u ; x_{m, i}(u)\right) \lambda_{m, i}(u)},
$$

we can use (2.4) and (2.6) to obtain (2.1) and (2.2).

We note that, for every weight $w$, the zeros of $p_{m}(w)$ interlace with those of $p_{m+1}(w)$; therefore, the natural extended interpolation of even degree is Lagrange interpolation with respect to the zeros of $\hat{p}_{2 m+1}=p_{m}(w) p_{m+1}(w)$.

Theorem 2.1 shows that this choice can be generalized to involve two different weights $w$ and $\bar{w}$. In the particular case $w(x)=\left(1-x^{2}\right)^{-1 / 2}$, we have $\bar{w}=\left(1-x^{2}\right)^{1 / 2}$ and we obtain the known result that the zeros of the Chebyshev polynomial of the second kind $U_{m}$ interlace with those of the Chebyshev polynomial of first kind $T_{m+1}$, i.e., $2 U_{m} T_{m+1}=U_{2 m+1}$.

The following theorem allows us to construct extended interpolation formulae of odd degree.

Theorem 2.3. If $w$ is any weight function and $w_{1}(x)=(1-x) w(x), w_{2}(x)=$ $(1+x) w(x)$, then the polynomial $V_{2 m}=p_{m}\left(w_{1}\right) p_{m}\left(w_{2}\right)$ satisfies

$$
\begin{aligned}
V_{2 m}^{\prime}\left(x_{m, i}\left(w_{1}\right)\right)=-D_{m}\left(1+x_{m, i}\left(w_{1}\right)\right)^{-1} \lambda_{m, i}^{-1}\left(w_{1}\right), & \\
& i=1, \ldots, m, m \in N,
\end{aligned}
$$




$$
\begin{aligned}
V_{2 m}^{\prime}\left(x_{m, i}\left(w_{2}\right)\right)=D_{m}\left(1-x_{m, i}\left(w_{2}\right)\right)^{-1} \lambda_{m, i}^{-1}\left(w_{2}\right), & \\
i & =1, \ldots, m, m \in N,
\end{aligned}
$$

where $D_{m}=\gamma_{m}\left(w_{2}\right) / \gamma_{m}\left(w_{1}\right)+\gamma_{m}\left(w_{1}\right) / \gamma_{m}\left(w_{2}\right)<\infty$.

Hence $p_{m}\left(w_{1}\right)$ and $p_{m}\left(w_{2}\right)$ have no common zeros and there holds

$$
x_{m, i}\left(w_{1}\right)<x_{m, i}\left(w_{2}\right), \quad i=1, \ldots, m, m \in N .
$$

Proof. Consider the Fourier expansion of $p_{m}\left(w_{1}\right)$ in the system $\left\{p_{k}(\bar{w})\right\}$ with $\bar{w}(x)=\left(1-x^{2}\right) w(x)$,

$$
p_{m}\left(w_{1} ; x\right)=\sum_{k=m-1}^{m} c_{k} p_{k}(\bar{w} ; x)
$$

where

$$
c_{k}=\int_{-1}^{1} p_{k}(\bar{w} ; x) p_{m}\left(w_{1} ; x\right) \bar{w}(x) d x, \quad k=m-1, m .
$$

In particular, $c_{m-1}=\gamma_{m-1}(\bar{w}) / \gamma_{m}\left(w_{1}\right)$ and $c_{m}=\gamma_{m}\left(w_{1}\right) / \gamma_{m}(\bar{w})$. Then, by (2.10),

$$
\begin{aligned}
p_{m}\left(w_{1} ; x_{m, i}\left(w_{2}\right)\right)= & \frac{\gamma_{m-1}(\bar{w})}{\gamma_{m}\left(w_{1}\right)} p_{m-1}\left(\bar{w} ; x_{m, i}\left(w_{2}\right)\right) \\
& +\frac{\gamma_{m}\left(w_{1}\right)}{\gamma_{m}(\bar{w})} p_{m}\left(\bar{w} ; x_{m, i}\left(w_{2}\right)\right) .
\end{aligned}
$$

Considering the Fourier expansions of $(1-x) p_{m}(\bar{w} ; x)$ and of $(1-x) p_{m-1}(\bar{w} ; x)$ in the system $\left\{p_{k}\left(w_{2}\right)\right\}$, we have by similar computations

$$
\begin{gathered}
\left(1-x_{m, i}\left(w_{2}\right)\right) p_{m}\left(\bar{w} ; x_{m, i}\left(w_{2}\right)\right)=\frac{\gamma_{m}(\bar{w}) \gamma_{m-1}\left(w_{2}\right)}{\gamma_{m}^{2}\left(w_{2}\right)} p_{m-1}\left(w_{2} ; x_{m, i}\left(w_{2}\right)\right), \\
\left(1-x_{m, i}\left(w_{2}\right)\right) p_{m-1}\left(\bar{w} ; x_{m, i}\left(w_{2}\right)\right)=\frac{\gamma_{m-1}\left(w_{2}\right)}{\gamma_{m-1}(\bar{w})} p_{m-1}\left(w_{2} ; x_{m, i}\left(w_{2}\right)\right) .
\end{gathered}
$$

These relations, together with $(2.11)$, give us

$$
\left(1-x_{m, i}\left(w_{2}\right)\right) p_{m}\left(w_{1} ; x_{m, i}\left(w_{2}\right)\right)=E_{m} p_{m-1}\left(w_{2} ; x_{m, i}\left(w_{2}\right)\right),
$$

with

$$
E_{m}=\gamma_{m-1}\left(w_{2}\right) / \gamma_{m}\left(w_{1}\right)+\gamma_{m}\left(w_{1}\right) \gamma_{m-1}\left(w_{2}\right) / \gamma_{m}^{2}\left(w_{2}\right)>0
$$

We finally consider the Fourier expansion of $p_{m}\left(w_{2}\right)$ in the system $\left\{p_{k}(\bar{w})\right\}$,

$$
p_{m}\left(w_{2} ; x\right)=\sum_{k=m-1}^{m} d_{k} p_{k}(\bar{w} ; x)
$$

where

$$
d_{k}=\int_{-1}^{1} p_{k}(\bar{w} ; x) p_{m}\left(w_{2} ; x\right) \bar{w}(x) d x, \quad k=m-1, m,
$$


and obtain $d_{m-1}=-\gamma_{m-1}(\bar{w}) / \gamma_{m}\left(w_{2}\right)$ and $d_{m}=\gamma_{m}\left(w_{2}\right) / \gamma_{m}(\bar{w})$. Then, by (2.13),

$$
\begin{aligned}
p_{m}\left(w_{2} ; x_{m, i}\left(w_{1}\right)\right)= & -\frac{\gamma_{m-1}(\bar{w})}{\gamma_{m}\left(w_{2}\right)} p_{m-1}\left(\bar{w} ; x_{m, i}\left(w_{1}\right)\right) \\
& +\frac{\gamma_{m}\left(w_{2}\right)}{\gamma_{m}(\bar{w})} p_{m}\left(\bar{w} ; x_{m, i}\left(w_{1}\right)\right) .
\end{aligned}
$$

Similarly,

$$
\begin{gathered}
\left(1+x_{m, i}\left(w_{1}\right)\right) p_{m}\left(\bar{w} ; x_{m, i}\left(w_{1}\right)\right)=-\frac{\gamma_{m}(\bar{w}) \gamma_{m-1}\left(w_{1}\right)}{\gamma_{m}^{2}\left(w_{1}\right)} p_{m-1}\left(w_{1} ; x_{m, i}\left(w_{1}\right)\right), \\
\left(1+x_{m, i}\left(w_{1}\right)\right) p_{m-1}\left(\bar{w} ; x_{m, i}\left(w_{1}\right)\right)=\frac{\gamma_{m-1}\left(w_{1}\right)}{\gamma_{m-1}(\bar{w})} p_{m-1}\left(w_{1} ; x_{m, i}\left(w_{1}\right)\right) .
\end{gathered}
$$

These relations, together with $(2.14)$, yield

$$
\left(1+x_{m, i}\left(w_{1}\right)\right) p_{m}\left(w_{2} ; x_{m, i}\left(w_{1}\right)\right)=-F_{m} p_{m-1}\left(w_{1} ; x_{m, i}\left(w_{1}\right)\right)
$$

with

$$
F_{m}=\gamma_{m-1}\left(w_{1}\right) / \gamma_{m}\left(w_{2}\right)+\gamma_{m}\left(w_{2}\right) \gamma_{m-1}\left(w_{1}\right) / \gamma_{m}^{2}\left(w_{1}\right)>0 .
$$

From (2.12) and (2.15) we conclude that the zeros of $p_{m}\left(w_{1}\right)$ are different from those of $p_{m}\left(w_{2}\right)$. Now, since

$$
\begin{aligned}
& V_{2 m}^{\prime}\left(x_{m, i}\left(w_{1}\right)\right)=p_{m}^{\prime}\left(w_{1} ; x_{m, i}\left(w_{1}\right)\right) p_{m}\left(w_{2} ; x_{m, i}\left(w_{1}\right)\right), \\
& V_{2 m}^{\prime}\left(x_{m, i}\left(w_{2}\right)\right)=p_{m}^{\prime}\left(w_{2} ; x_{m, i}\left(w_{2}\right)\right) p_{m}\left(w_{1} ; x_{m, i}\left(w_{2}\right)\right),
\end{aligned}
$$

recalling (2.7), we use (2.12) and (2.15) to obtain (2.8) and (2.9). From Theorem 2.1 one obtains the boundedness of $D_{m}$.

Remark. For our purposes it was sufficient to prove Theorems 2.2 and 2.3, assuming the measure $d \mu$ absolutely continuous; however by Theorem 2.1 , Theorems 2.2 and 2.3 are true for every measure $d \mu \in M$.

In the particular case $w(x)=\left(1-x^{2}\right)^{-1 / 2}$, we have

$$
w_{1}(x)=(1-x)^{1 / 2}(1+x)^{-1 / 2}, \quad w_{2}=w_{1}^{-1}
$$

and we obtain the well-known separation property given by the identity

$$
p_{m}\left(w_{1}\right) p_{m}\left(w_{1}^{-1}\right)=k_{m}^{2} U_{2 m}, \quad k_{m}=\frac{1 \cdot 3 \cdot \cdots \cdot(2 m-1)}{2 \cdot 4 \cdot \cdots \cdot(2 m)}
$$

The preceding theorems assure us that the polynomials $p_{m}(\bar{w})$ and $p_{m+1}(w)$, as well as $p_{m}\left(w_{1}\right)$ and $p_{m}\left(w_{2}\right)$, have no common zeros; thus, it is possible to construct extended interpolation rules on their zeros. 
We first consider the extended interpolation polynomial $L_{2 m+1}(w, \bar{w} ; f)$ on the zeros $t_{2 m+1, i}, i=1, \ldots, 2 m+1$, of $Q_{2 m+1}=p_{m}(\bar{w}) p_{m+1}(w)$,

$$
\begin{aligned}
L_{2 m+1}(w, \bar{w} ; f ; x)= & \sum_{i=1}^{2 m+1} \frac{Q_{2 m+1}(x)}{Q_{2 m+1}^{\prime}\left(t_{2 m+1, i}\right)\left(x-t_{2 m+1, i}\right)} f\left(t_{2 m+1, i}\right) \\
= & \sum_{i=1}^{m+1} \frac{Q_{2 m+1}(x)}{Q_{2 m+1}^{\prime}\left(x_{m+1, i}(w)\right)\left(x-x_{m+1, i}(w)\right)} f\left(x_{m+1, i}(w)\right) \\
& +\sum_{i=1}^{m} \frac{Q_{2 m+1}(x)}{Q_{2 m+1}^{\prime}\left(x_{m, i}(\bar{w})\right)\left(x-x_{m, i}(\bar{w})\right)} f\left(x_{m, i}(\bar{w})\right) .
\end{aligned}
$$

Recalling (2.1) and (2.2), we can thus write

$$
\begin{aligned}
& L_{2 m+1}(w, \bar{w} ; f ; x)= C_{m}^{-1} p_{m+1}(w ; x) p_{m}(\bar{w} ; x) \\
& \times\left\{\sum_{i=1}^{m+1} \frac{\lambda_{m+1, i}(w)\left(1-x_{m+1, i}^{2}(w)\right)}{x-x_{m+1, i}(w)} f\left(x_{m+1, i}(w)\right)\right. \\
&\left.-\sum_{i=1}^{m} \frac{\lambda_{m, i}(\bar{w})}{x-x_{m, i}(\bar{w})} f\left(x_{m, i}(\bar{w})\right)\right\},
\end{aligned}
$$

where $C_{m}=\gamma_{m}(\bar{w}) / \gamma_{m+1}(w)+\gamma_{m+1}(w) / \gamma_{m}(\bar{w})$.

Similarly, by (2.8) and (2.9), the extended interpolation polynomial $L_{2 m}\left(w_{1}, w_{2} ; f\right)$ on the zeros of $V_{2 m}=p_{m}\left(w_{1}\right) p_{m}\left(w_{2}\right)$ takes on the form

$$
\begin{aligned}
L_{2 m}\left(w_{1}, w_{2} ; f ; x\right)= & D_{m}^{-1} p_{m}\left(w_{1} ; x\right) p_{m}\left(w_{2} ; x\right) \\
& \times\left\{\sum_{i=1}^{m} \lambda_{m, i}\left(w_{2}\right) \frac{1-x_{m, i}\left(w_{2}\right)}{x-x_{m, i}\left(w_{2}\right)} f\left(x_{m, i}\left(w_{2}\right)\right)\right. \\
& \left.\quad-\sum_{i=1}^{m} \lambda_{m, i}\left(w_{1}\right) \frac{1+x_{m, i}\left(w_{1}\right)}{x-x_{m, i}\left(w_{1}\right)} f\left(x_{m, i}\left(w_{1}\right)\right)\right\},
\end{aligned}
$$

where $D_{m}=\gamma_{m}\left(w_{2}\right) / \gamma_{m}\left(w_{1}\right)+\gamma_{m}\left(w_{1}\right) / \gamma_{m}\left(w_{2}\right)$.

Since, for any weight $w$, the fundamental Lagrange polynomials are

$$
l_{n, i}(w ; x)=\frac{\gamma_{n-1}(w)}{\gamma_{n}(w)} \lambda_{n, i}(w) p_{n-1}\left(w ; x_{n, i}(w)\right) \frac{\left.p_{n}(w) x\right)}{x-x_{n, i}(w)},
$$

by the recurrence formula of $p_{n}(w)$, we can write the extended interpolation polynomial $L_{2 m+1}(w, w ; f)$ on the zeros of $\hat{p}_{2 m+1}=p_{m}(w) p_{m+1}(w)$ as

$$
\begin{aligned}
L_{2 m+1}(w, w ; f ; x)= & \frac{\gamma_{m}(w)}{\gamma_{m+1}(w)} p_{m}(w ; x) p_{m+1}(w ; x) \\
& \times\left\{\sum_{i=1}^{m+1} \frac{\lambda_{m+1, i}(w)}{x-x_{m+1, i}(w)} f\left(x_{m+1, i}(w)\right)\right. \\
& \left.\quad-\sum_{i=1}^{m} \frac{\lambda_{m, i}(w)}{x-x_{m, i}(w)} f\left(x_{m, i}(w)\right)\right\} .
\end{aligned}
$$


Furthermore, if $r$ and $s$ are nonnegative integers, we may consider the extended quasi-Lagrange interpolation polynomial on the zeros of $Q_{2 m+1}=$ $p_{m}(\bar{w}) p_{m+1}(w)$ and on the points \pm 1 . For the definition of quasi-Lagrange interpolation polynomimal see [11]. Recalling (2.1) and (2.2), we deduce that this is the polynomial of degree $2 m+r+s$ represented in the form

$$
\begin{aligned}
L_{2 m+1}^{(r, s)}(w, \bar{w} ; f ; x) & \\
= & C_{m}^{-1}(1-x)^{r}(1+x)^{s} p_{m+1}(w ; x) p_{m}(\bar{w} ; x) \\
& \times\left\{\sum_{i=1}^{m+1} \frac{\lambda_{m+1, i}(w) f\left(x_{m+1, i}(w)\right)}{\left(x-x_{m+1, i}(w)\right)\left(1-x_{m+1, i}(w)\right)^{r-1}\left(1+x_{m+1, i}(w)\right)^{s-1}}\right. \\
& \left.-\sum_{i=1}^{m} \frac{\lambda_{m, i}(\bar{w}) f\left(x_{m, i}(\bar{w})\right)}{\left(x-x_{m, i}(\bar{w})\right)\left(1-x_{m, i}(\bar{w})\right)^{r}\left(1+x_{m, i}(\bar{w})\right)^{s}}\right\} \\
& +h_{1}(x) f(-1)+h_{2}(x) f(1),
\end{aligned}
$$

where

$$
\begin{aligned}
h_{1}(x)= & (1+x)^{s} p_{m}(\bar{w} ; x) p_{m+1}(w ; x) \\
& \times \sum_{j=0}^{r-1}(-1)^{j} \frac{1}{j !}\left[\frac{1}{p_{m}(\bar{w} ; t) p_{m+1}(w ; t)(1+t)^{s}}\right]_{t=1}^{(j)}(1-x)^{j}, \\
h_{2}(x)= & (1-x)^{r} p_{m}(\bar{w} ; x) p_{m+1}(w ; x) \\
& \times \sum_{j=0}^{s-1} \frac{1}{j !}\left[\frac{1}{p_{m}(\bar{w} ; t) p_{m+1}(w ; t)(1-t)^{r}}\right]_{t=-1}^{(j)}(1+x)^{j} .
\end{aligned}
$$

Similarly, by (2.8) and (2.9), the extended quasi-Lagrange interpolation polynomial on the zeros of $V_{2 m}=p_{m}\left(w_{1}\right) p_{m}\left(w_{2}\right)$ and on the points \pm 1 can be written in the form

$$
\begin{aligned}
L_{2 m}^{(r, s)}( & \left.w_{1}, w_{2} ; f ; x\right) \\
= & D_{m}^{-1}(1-x)^{r}(1+x)^{s} p_{m}\left(w_{1} ; x\right) p_{m}\left(w_{2} ; x\right) \\
& \times\left\{\sum_{i=1}^{m} \frac{\lambda_{m, i}\left(w_{2}\right) f\left(x_{m, i}\left(w_{2}\right)\right)}{\left(x-x_{m, i}\left(w_{2}\right)\right)\left(1-x_{m, i}\left(w_{2}\right)\right)^{r-1}\left(1+x_{m, i}\left(w_{2}\right)\right)^{s}}\right. \\
& \left.-\sum_{i=1}^{m} \frac{\lambda_{m, i}\left(w_{1}\right) f\left(x_{m, i}\left(w_{1}\right)\right)}{\left(x-x_{m, i}\left(w_{1}\right)\right)\left(1-x_{m, i}\left(w_{1}\right)\right)^{r}\left(1+x_{m, i}\left(w_{1}\right)\right)^{s-1}}\right\} \\
& +k_{1}(x) f(-1)+k_{2}(x) f(1),
\end{aligned}
$$

where $k_{1}$ and $k_{2}$ have the same expressions as $h_{1}$ and $h_{2}$, with $w$ and $\bar{w}$ replaced by $w_{1}$ and $w_{2}$, respectively. 
Finally, we consider the extended quasi-Lagrange interpolation polynomial on the zeros of $\hat{p}_{2 m+1}=p_{m}(w) p_{m+1}(w)$ and on the points \pm 1 ,

$$
\begin{aligned}
L_{2 m+1}^{(r, s)}(w, w ; f ; x) & \\
= & \frac{\gamma_{m}(w)}{\gamma_{m+1}(w)}(1-x)^{r}(1+x)^{s} p_{m+1}(w ; x) p_{m}(w ; x) \\
& \times\left\{\sum_{i=1}^{m+1} \frac{\lambda_{m+1, i}(w) f\left(x_{m+1, i}(w)\right)}{\left(x-x_{m+1, i}(w)\right)\left(1-x_{m+1, i}(w)\right)^{r}\left(1+x_{m+1, i}(w)\right)^{s}}\right. \\
& \left.-\sum_{i=1}^{m} \frac{\lambda_{m, i}(w) f\left(x_{m, i}(w)\right)}{\left(x-x_{m, i}(w)\right)\left(1-x_{m, i}(w)\right)^{r}\left(1+x_{m, i}(w)\right)^{s}}\right\}
\end{aligned}
$$

where $\hat{h}_{1}$ and $\hat{h}_{2}$ have the same expressions as $h_{1}$ and $h_{2}$, with $\bar{w}$ replaced by $w$.

As we have already said in the introduction, our formulae are simpler than the corresponding ones for ordinary extended interpolation, and also for ordinary interpolation (cf. (1.1)). Indeed, the terms $p_{m}\left(w ; x_{m+1, i}(w)\right)$ and $p_{m-1}\left(\bar{w} ; x_{m, i}(\bar{w})\right)$ are not present in the two sums of $(2.16)$; moreover, each sum is independent of the zeros that appear in the other. This facilitates the study of convergence.

\section{ON THE Distribution OF THE ZEROS OF $Q_{2 m+1}$ AND $V_{2 m}$}

Theorems 2.2 and 2.3 assure us that for any weight $w$ the zeros of $Q_{2 m+1}$ and $V_{2 m}$ are different from one another and, furthermore, they all belong to $(-1,1)$. However, if we assume that $w$ is a generalized smooth Jacobi weight $(w \in G S J)$, then we can obtain more precise results. Such a weight is defined by

$$
w(x)=\varphi(x)(1-x)^{\alpha} \prod_{k=1}^{n}\left|x-t_{k}\right|^{\gamma_{k}}(1+x)^{\beta}, \quad-1 \leq x \leq 1,
$$

where $\alpha, \beta, \gamma_{k}>-1, k=1, \ldots, n,-1<t_{1}<t_{2}<\cdots<t_{n}<1$, and $0<\varphi \in D T:=\left\{g \in C[-1,1] \mid \int_{-1}^{1} \omega(g ; \delta) \delta^{-1} d \delta<\infty\right\}$; here $\omega$ denotes the usual modulus of continuity.

We now prove the following

Theorem 3.1. If $w \in G S J$ and $\bar{w}=\left(1-x^{2}\right) w(x)$, then the zeros $t_{2 m+1, i}=$ $\cos \theta_{2 m+1, i}, i=1, \ldots, 2 m+1$, of $Q_{2 m+1}=p_{m+1}(w) p_{m}(\bar{w})$, in natural order, satisfy

$$
\theta_{2 m+1, i}-\theta_{2 m+1, i+1} \sim m^{-1,1}
$$

uniformly in $1 \leq i \leq 2 m, m \in N$.

\footnotetext{
'If $A$ and $B$ are two expressions depending on some variables, then we write $A \sim B$ if and only if $\left|A B^{-1}\right| \leq$ const and $\left|A^{-1} B\right| \leq$ const, uniformly for the variables in question.
} 
Proof. From Theorem 2.2 we have

$$
x_{m+1, i}(w)<x_{m, i}(\bar{w})<x_{m+1, i+1}(w), \quad i=1,2, \ldots, m .
$$

Therefore, in order to prove (3.1), it is sufficient to show that

$$
x_{m, i}(\bar{w})-x_{m+1, i}(w) \sim \frac{\sqrt{1-x^{2}}}{m} \sim x_{m+1, i+1}(w)-x_{m, i}(\bar{w}),
$$

uniformly in $1 \leq i \leq m, m \in N$ and $x \in\left[x_{m+1, i}(w), x_{m+1, i+1}(w)\right]$.

We prove the first equivalence of (3.2) by using a technique used already in [3] and suggested by Nevai. We recall that the fundamental Lagrange polynomials $l_{m, i}(w)$ can be written as

$$
l_{m+1, i}(w ; x)=\frac{\gamma_{m}(w)}{\gamma_{m+1}(w)} \lambda_{m+1, i}(w) p_{m}\left(w ; x_{m+1, i}(w)\right) \frac{p_{m+1}(w ; x)}{x-x_{m+1, i}(w)}
$$

Moreover, it is well known that

$$
\left|l_{m+1, i}(w ; x)\right| \sim 1, \quad x \in\left[x_{m+1, i}(w), x_{m+1, i+1}(w)\right]
$$

(see [11]). Thus by (3.3), with $x=x_{m, i}(\bar{w}) \in\left[x_{m+1, i}(w), x_{m+1, i+1}(w)\right]$, we conclude

$$
1 \sim \frac{\gamma_{m}(w)}{\gamma_{m+1}(w)} \lambda_{m+1, i}(w)\left|p_{m}\left(w ; x_{m+1, i}(w)\right)\right| \frac{\left|p_{m+1}\left(w ; x_{m, i}(\bar{w})\right)\right|}{\left|x_{m, i}(\bar{w})-x_{m+1, i}(w)\right|} .
$$

Then by (2.6), and since

we have

$$
\frac{\gamma_{m}(w)}{\gamma_{m+1}(w)} B_{m} \sim 1
$$

$1 \sim \lambda_{m+1, i}(w)\left|p_{m}\left(w ; x_{m+1, i}(w)\right)\right|\left|p_{m-1}\left(\bar{w} ; x_{m, i}(\bar{w})\right)\right|\left|x_{m, i}(\bar{w})-x_{m+1, i}(w)\right|^{-1}$.

Since $w, \bar{w} \in G S J$, the relations

$$
\begin{gathered}
\lambda_{m}(w ; x) \sim m^{-1} w_{m}^{*}(x), \\
w_{m}^{*}\left(x_{m, i}(w)\right) p_{m-1}^{2}\left(w ; x_{m, i}(w)\right) \sim 1-x_{m, i}^{2}(w),
\end{gathered}
$$

where

$$
w_{m}^{*}(x)=\left(\sqrt{1-x}+m^{-1}\right)^{2 \alpha+1} \prod_{k=1}^{n}\left(\left|t_{k}-x\right|+m^{-1}\right)^{\gamma_{k}}\left(\sqrt{1+x}+m^{-1}\right)^{2 \beta+1}
$$

(see [11]), taking into account that

$$
x_{m+1, i}(w) \sim x \sim x_{m, i}(\bar{w}) \text { for } x \in\left[x_{m+1, i}(w), x_{m, i}(\bar{w})\right],
$$

imply

$$
x_{m, i}(\bar{w})-x_{m+1, i}(w) \sim m^{-1} \frac{\left(1-x_{m+1, i}(w)\right)^{\alpha / 2+3 / 4}\left(1+x_{m+1, i}(w)\right)^{\beta / 2+3 / 4}}{\left(1-x_{m, i}(\bar{w})\right)^{\alpha / 2+1 / 4}\left(1+x_{m, i}(\bar{w})\right)^{\beta / 2+1 / 4}} .
$$

This relation implies immediately the first equivalence of (3.2). The second is proved similarly.

We omit the proof of the following theorem, since it is very similar to that of the previous one. 
Theorem 3.2. If $w \in G S J$ and $w_{1}(x)=(1-x) w(x), w_{2}(x)=(1+x) w(x)$, then the zeros $z_{2 m, i}=\cos \sigma_{2 m, i}, i=1, \ldots, 2 m$, of $V_{2 m}=p_{m}\left(w_{1}\right) p_{m}\left(w_{2}\right)$, in natural order, satisfy

$$
\sigma_{2 m, i}-\sigma_{2 m, i+1} \sim m^{-1}
$$

uniformly in $1 \leq i \leq 2 m-1, m \in N$.

Remark. Theorems 3.1 and 3.2 are not needed to study convergence of the extended interpolation formulae (2.16), (2.17), (2.19), and (2.20). We have stated them here, since they may be useful for finding the numerical solution of singular integral equations by collocation methods. Indeed, the integrals (in the Cauchy principal value sense or weakly singular) which appear in the equation, are often treated by two different interpolations (one corresponding to the collocation and another to the quadrature). In order to avoid divergence and numerical cancellation, it is necessary that the collocation points and the quadrature knots are not only different from one another, but also sufficiently far apart.

\section{UNIFORM CONVERGENCE OF EXTENDED INTERPOLATION}

We start with some preliminary remarks, assuming $w \in G S J$ throughout. For any $x \in(-1,1), m \in N$, we denote by $x_{c(m)}(w)=x_{m, c}(w)$ the knot closest to $x$, defined by

$$
x_{m, c}(w)= \begin{cases}x_{m, d}(w) & \text { if } x-x_{m, d}(w) \leq x_{m, d+1}(w)-x, \\ x_{m, d+1}(w) & \text { if } x-x_{m, d}(w)>x_{m, d+1}(w)-x,\end{cases}
$$

where $x_{m, d}(w) \leq x \leq x_{m, d+1}(w)$ for some $d \in\{0,1, \ldots, m\}$ and $x_{m, 0}(w)$ $=-1, x_{m, m+1}(w)=1$. By Theorems 3.1 and 3.2, and recalling (3.4), we find

$$
\begin{gathered}
\lambda_{m+1, i}(w)\left(1-x_{m+1, i}(w)\right)^{-r+1}\left(1+x_{m+1, i}(w)\right)^{-s+1} \\
\sim \lambda_{m, i}(\bar{w})\left(1-x_{m, i}(\bar{w})\right)^{-r}\left(1+x_{m, i}(\bar{w})\right)^{-s}, \\
\lambda_{m, i}\left(w_{1}\right)\left(1-x_{m, i}\left(w_{1}\right)\right)^{-r}\left(1+x_{m, i}\left(w_{1}\right)\right)^{-s} \\
\sim \lambda_{m, i}\left(w_{2}\right)\left(1-x_{m, i}\left(w_{2}\right)\right)^{-r+1}\left(1+x_{m, i}\left(w_{2}\right)\right)^{-s+1},
\end{gathered}
$$

where $\bar{w}(x)=\left(1-x^{2}\right) w(x), w_{1}(x)=(1-x) w(x), w_{2}(x)=(1+x) w(x)$, and $r, s \in N$. The equivalences (3.5), (4.1), (4.2) and the inequality

$$
\left|p_{m}(w ; x)\right| \leq \text { const } w_{m}^{*}(x)^{-1 / 2}
$$

where $w_{m}^{*}$ is defined by (3.6) (see [11]), allow us to write

$$
\begin{aligned}
& \left|p_{m}(\bar{w} ; x) p_{m+1}(w ; x)\right| \frac{\lambda_{m, c}(\bar{w})}{\left|x-x_{m, c}(\bar{w})\right|} \sim 1, \\
& \left|p_{m}\left(w_{1} ; x\right) p_{m}\left(w_{2} ; x\right)\right| \frac{\lambda_{m, c}\left(w_{1}\right)}{\left|x-x_{m, c}\left(w_{1}\right)\right|} \sim 1 .
\end{aligned}
$$

The following lemmas are needed to prove the subsequent results. 
Lemma 4.1. Let $r, i, m$ be positive integers with $m \geq \max \{4(r+1), r+i\}$. Given any function $f \in C^{r}[-1,1]$, there exists a polynomial $q_{m}$ of degree $m$ such that for $x \in[-1,1]$

$$
\begin{aligned}
q_{m}^{(k)}( \pm 1)= & f^{(k)}( \pm 1), \quad k=0,1, \ldots, r \\
\left|q_{m}^{(k)}(x)-f_{m}^{(k)}(x)\right| \leq \text { const } & {\left[\frac{\sqrt{1-x^{2}}}{m}+\frac{1}{m^{2}}\right]^{r-k} } \\
& \times \omega_{i}\left(f^{(r)} ; \frac{\sqrt{1-x^{2}}}{m}+\frac{1}{m^{2}}\right), \quad k=0,1, \ldots, r, \\
\left|q_{m}^{(k)}(x)-f_{m}^{(k)}(x)\right| \leq & \text { const }\left[\frac{\sqrt{1-x^{2}}}{m}\right]^{r-k} \\
& \times \omega_{i}\left(f^{(r)} ; \frac{\sqrt{1-x^{2}}}{m}\right), \quad k=0,1, \ldots, r-i,
\end{aligned}
$$

where $\omega_{i}(g ; \delta)=\operatorname{Sup}_{0 \leq h \leq \delta}\left\|\Delta_{h}^{i} g\right\|_{[-1,1-i h]}, \delta>0$, is the ith modulus of continuity.

This lemma follows from Satz 4.2, 5.4, and 5.5 in [7].

Defining the functions

$$
\begin{gathered}
S_{m}^{\sigma}(w ; 1 ; x)=\sum_{\substack{i=1 \\
i \neq c}}^{m} \frac{\left(1-x_{m, i}(w)\right)^{\sigma}}{m\left|x-x_{m, i}(w)\right|}, \\
S_{m}^{\delta}(w ;-1 ; x)=\sum_{\substack{i=1 \\
i \neq c}}^{m} \frac{\left(1+x_{m, i}(w)\right)^{\delta}}{m\left|x-x_{m, i}(w)\right|}, \\
S_{m}^{\gamma}(w ; \tau ; x)=\sum_{\substack{i=1 \\
i \neq c}}^{m} \frac{\left(\left|\tau-x_{m, i}(w)\right|+m^{-1}\right)^{\gamma}}{m\left|x-x_{m, i}(w)\right|},
\end{gathered}
$$

where $c$ denotes the index corresponding to the closest knot to $x, \tau$ is a fixed point belonging to $(-1,1)$, and $\sigma, \delta, \gamma$ are real numbers, we also have the following lemmas. The proof of these results can be found in [4].

Lemma 4.2. If $w \in G S J$, then for every $x \in[-1,1]$

$$
S_{m}^{\sigma}(w ; 1 ; x) \leq \mathrm{const} \begin{cases}m^{-2 \sigma-1}\left(\sqrt{1-x}+m^{-1}\right)^{-2}+\left(\sqrt{1-x}+m^{-1}\right)^{2 \sigma-1} \log m \\ & \text { if } \sigma<-1 / 2, \\ \left(\sqrt{1-x}+m^{-1}\right)^{2 \sigma-1} \log m & \text { if }-1 / 2 \leq \sigma \leq 1 / 2, \\ \left(\sqrt{1-x}+m^{-1}\right)^{2 \sigma-1} \log m+1 & \text { if } \sigma>1 / 2 .\end{cases}
$$


Lemma 4.3. If $w \in G S J$, then for every $x \in[-1,1]$

$S_{m}^{\delta}(w ;-1 ; x) \leq \mathrm{const} \begin{cases}m^{-2 \delta-1}\left(\sqrt{1+x}+m^{-1}\right)^{-2}+\left(\sqrt{1+x}+m^{-1}\right)^{2 \delta-1} \log m \\ \left(\sqrt{1+x}+m^{-1}\right)^{2 \delta-1} \log m & \text { if } \delta<-1 / 2, \\ \left(\sqrt{1+x}+m^{-1}\right)^{2 \delta-1} \log m+1 & \text { if } \delta>1 / 2 \leq \delta \leq 1 / 2,\end{cases}$

Lemma 4.4. If $w \in G S J$, then for every $x \in[-1,1]$

$$
S_{m}^{\gamma}(w ; \tau ; x) \leq \mathrm{const} \begin{cases}\log m & \text { if } \gamma \geq 0, \\ 1+m^{-\gamma} & \text { if } \gamma<0 .\end{cases}
$$

For the sake of brevity, we shall prove the following theorems only for $w(x)=u^{\alpha, \beta}(x)=(1-x)^{\alpha}(1+x)^{\beta}, \alpha, \beta>-1$; but the extension to the more general case $w \in G S J$ is very easy.

Theorem 4.1. Let $w=u^{\alpha, \beta}, \alpha, \beta>-1$. For any function $f \in C^{s}[-1,1]$, $s \geq 0$, we have

$$
\begin{aligned}
\mid f(x)- & L_{2 m+1}(w, \bar{w} ; f ; x) \mid \\
\leq \text { const }[\log m & +\left(\sqrt{1-x}+m^{-1}\right)^{-2 \alpha-2} \\
& \left.+\left(\sqrt{1+x}+m^{-1}\right)^{-2 \beta-2}\right] \omega_{s}\left(f ; m^{-1}\right), \quad|x| \leq 1,
\end{aligned}
$$

where const is independent of $f$ and $m$.

Proof. Let $q_{m}$ be the polynomial defined by Lemma 4.1 corresponding to the function $f$. Then by (2.16),

$$
\begin{aligned}
\mid f(x) & -L_{2 m+1}(w, \bar{w} ; f ; x) \mid \\
\leq & \left|f(x)-q_{m}(x)\right|+\left|L_{2 m+1}\left(w, \bar{w} ; f-q_{m} ; x\right)\right| \\
\leq & \operatorname{const} \omega_{s}\left(f ; m^{-1}\right) \\
& \times\left\{1+\left|p_{m+1}(w ; x) p_{m}(\bar{w} ; x)\right|\left[\sum_{i=1}^{m+1} \frac{\lambda_{m+1, i}(w)\left(1-x_{m+1, i}^{2}(w)\right)}{\left|x-x_{m+1, i}(w)\right|}\right.\right. \\
& \left.\left.+\sum_{i=1}^{m} \frac{\lambda_{m, i}(\bar{w})}{\left|x-x_{m, i}(\bar{w})\right|}\right]\right\} .
\end{aligned}
$$

Recalling (4.1), (4.3), and (4.4), we obtain

$$
\begin{aligned}
& \left(\sqrt{1-x}+m^{-1}\right)^{2 \alpha+2}\left(\sqrt{1+x}+m^{-1}\right)^{2 \beta+2}\left|f(x)-L_{2 m+1}(w, \bar{w} ; f ; x)\right| \\
& \leq \operatorname{const} \omega_{s}\left(f ; m^{-1}\right) \sum_{\substack{i=1 \\
i \neq c}}^{m} \frac{\lambda_{m, i}(\bar{w})}{\left|x-x_{m, i}(\bar{w})\right|} \\
& \sim \operatorname{const} \omega_{s}\left(f ; m^{-1}\right) \sum_{\substack{i=1 \\
i \neq c}}^{m} \frac{\left(1-x_{m, i}(\bar{w})\right)^{\alpha+3 / 2}\left(1+x_{m, i}(\bar{w})\right)^{\beta+3 / 2}}{m\left|x-x_{m, i}(\bar{w})\right|} \\
& =: \text { const } \omega_{s}\left(f ; m^{-1}\right) s_{m}(x),
\end{aligned}
$$

where $c$ is the index corresponding to the closest knot to $x$. 
Since

$$
\begin{aligned}
s_{m}(x) \leq & (1+x)^{\beta+3 / 2} \sum_{i<c} \frac{\left(1-x_{m, i}(\bar{w})\right)^{\alpha+3 / 2}}{m\left(x-x_{m, i}(\bar{w})\right)} \\
& +(1-x)^{\alpha+3 / 2} \sum_{i>c} \frac{\left(1+x_{m, i}(\bar{w})\right)^{\beta+3 / 2}}{m\left(x_{m, i}(\bar{w})-x\right)} \\
\leq & \left(\sqrt{1+x}+m^{-1}\right)^{2 \beta+3} \sum_{i<c} \frac{\left(1-x_{m, i}(\bar{w})\right)^{\alpha+3 / 2}}{m\left(x-x_{m, i}(\bar{w})\right)} \\
& +\left(\sqrt{1-x}+m^{-1}\right)^{2 \alpha+3} \sum_{i>c} \frac{\left(1+x_{m, i}(\bar{w})\right)^{\beta+3 / 2}}{m\left(x_{m, i}(\bar{w})-x\right)}
\end{aligned}
$$

and applying Lemmas 4.2 and 4.3 with $\sigma, \delta>1 / 2$, respectively, we have

$$
\begin{aligned}
s_{m}(x) \leq & \left(\sqrt{1+x}+m^{-1}\right)^{2 \beta+3}\left[\left(\sqrt{1-x}+m^{-1}\right)^{2 \alpha+2} \log m+1\right] \\
& +\left(\sqrt{1-x}+m^{-1}\right)^{2 \alpha+3}\left[\left(\sqrt{1+x}+m^{-1}\right)^{2 \beta+2} \log m+1\right] .
\end{aligned}
$$

Combining this last inequality with (4.7), we obtain (4.6).

We note that inequality (4.6) improves a result obtained in a different way in [2, Corollary 2]. The following theorem shows that the extended interpolation polynomial $L_{2 m+1}^{(1,1)}(w, \bar{w} ; f)$ has a better behavior.

Theorem 4.2. Let $w=u^{\alpha, \beta}, \alpha, \beta>-1$. For any $f \in C^{s}[-1,1], s \geq 0$, we have

$$
\begin{aligned}
& \left|f(x)-L_{2 m+1}^{(1,1)}(w, \bar{w} ; f ; x)\right| \\
& \leq \text { const } \log m\left[\left(\sqrt{1-x}+m^{-1}\right)^{-2 \alpha}\right. \\
& \left.\quad+\left(\sqrt{1+x}+m^{-1}\right)^{-2 \beta}\right] \omega_{s}\left(f ; m^{-1}\right), \quad|x| \leq 1,
\end{aligned}
$$

where const is independent of $f$ and $m$.

Proof. Let $q_{m}$ be the polynomial defined by Lemma 4.1 corresponding to the function $f$; thus, $q_{m}( \pm 1)=f( \pm 1)$.

Recalling (4.1), (4.3), and (4.4), and proceeding as in the proof of the Theorem 4.1 , we find

$$
\begin{aligned}
& \left(\sqrt{1-x}+m^{-1}\right)^{2 \alpha}\left(\sqrt{1+x}+m^{-1}\right)^{2 \beta}\left|L_{2 m+1}^{(1,1)}(w, \bar{w} ; f ; x)-f(x)\right| \\
& \quad \leq \operatorname{const} \omega_{s}\left(f ; m^{-1}\right) \sum_{\substack{i=1 \\
i \neq c}}^{m} \frac{\lambda_{m, i}(\bar{w})}{\left(1-x_{m, i}^{2}(\bar{w})\right)\left|x-x_{m, i}(\bar{w})\right|} \\
& \quad \sim \operatorname{const} \omega_{s}\left(f ; m^{-1}\right) \sum_{\substack{i=1 \\
i \neq c}}^{m} \frac{\left(1-x_{m, i}(\bar{w})\right)^{\alpha+1 / 2}\left(1+x_{m, i}(\bar{w})\right)^{\beta+1 / 2}}{m\left|x-x_{m, i}(\bar{w})\right|} \\
& \quad=\text { const } \omega_{s}\left(f ; m^{-1}\right) S_{m}(x) .
\end{aligned}
$$


Assuming that $\alpha, \beta \leq 0$, we have

$$
\begin{aligned}
S_{m}(x) \leq & \left(\sqrt{1-x}+m^{-1}\right)^{2 \alpha} \sum_{\substack{i=1 \\
i \neq c}}^{m} \frac{\left(1+x_{m, i}(\bar{w})\right)^{\beta+1 / 2}}{m\left|x-x_{m, i}(\bar{w})\right|} \\
& +\left(\sqrt{1+x}+m^{-1}\right)^{2 \beta} \sum_{\substack{i=1 \\
i \neq c}}^{m} \frac{\left(1-x_{m, i}(\bar{w})\right)^{\alpha+1 / 2}}{m\left|x-x_{m, i}(\bar{w})\right|} .
\end{aligned}
$$

Thus, applying Lemmas 4.2 and 4.3 , we obtain (4.8).

On the other hand, if $\alpha<0$ and $\beta>0$, then

$$
S_{m}(x) \leq\left(\sqrt{1-x}+m^{-1}\right)^{2 \alpha} \sum_{\substack{i=1 \\ i \neq c}}^{m} \frac{\left(1+x_{m, i}(\bar{w})\right)^{\beta+1 / 2}}{m\left|x-x_{m, i}(\bar{w})\right|}+\sum_{\substack{i=1 \\ i \neq c}}^{m} \frac{\left(1-x_{m, i}(\bar{w})\right)^{\alpha+1 / 2}}{m\left|x-x_{m, i}(\bar{w})\right|} .
$$

Applying Lemmas 4.2 and 4.3, we deduce (4.8). Finally, if $\alpha>0$ and $\beta<0$, or $\alpha>0$ and $\beta>0$, then (4.8) follows again.

Inequality (4.8) improves two results obtained in a different way in [2, Corollaries 1, 3].

We omit the proof of the following theorem, since it is very similar to the proofs of Theorems 4.1 and 4.2, making use of inequalities (4.2) and (4.5) instead of (4.1) and (4.4).

Theorem 4.3. Let $w=u^{\alpha, \beta}, \alpha, \beta>-1$. For any function $f \in C^{s}[-1,1]$, $s \geq 0$,

$$
\begin{aligned}
& \left|f(x)-L_{2 m}\left(w_{1}, w_{2} ; f ; x\right)\right| \\
& \leq \text { const }\left[\log m+\left(\sqrt{1-x}+m^{-1}\right)^{-2 \alpha-2}\right. \\
& \left.+\left(\sqrt{1+x}+m^{-1}\right)^{-2 \beta-2}\right] \omega_{s}\left(f ; m^{-1}\right), \quad|x| \leq 1, \\
& \left|f(x)-L_{2 m}^{(1,1)}\left(w_{1}, w_{2} ; f ; x\right)\right| \\
& \leq \text { const } \log m\left[\left(\sqrt{1-x}+m^{-1}\right)^{-2 \alpha}\right. \\
& \left.\quad+\left(\sqrt{1+x}+m^{-1}\right)^{-2 \beta}\right] \omega_{s}\left(f ; m^{-1}\right), \quad|x| \leq 1,
\end{aligned}
$$

where const is independent of $f$ and $m$.

Finally, the following theorem exhibits the behavior of the interpolation formulae (2.18) and (2.21).

Theorem 4.4. Let $w=u^{\alpha, \beta}, \alpha, \beta>-1$. For any function $f \in C^{s}[-1,1]$, $s \geq 0$, we have

$$
\begin{aligned}
& \left|f(x)-L_{2 m+1}(w, w ; f ; x)\right| \\
& \leq \text { const } \omega_{s}\left(f ; m^{-1}\right)\left[\frac{\log m+\left(\sqrt{1-x}+m^{-1}\right)^{-2 \alpha}}{\sqrt{1-x}+m^{-1}}\right. \\
& \left.\quad+\frac{\log m+\left(\sqrt{1+x}+m^{-1}\right)^{-2 \beta}}{\sqrt{1+x}+m^{-1}}\right], \quad|x| \leq 1,
\end{aligned}
$$




$$
\begin{aligned}
& \quad \begin{array}{l}
\left|f(x)-L_{2 m+1}^{(1,1)}(w, w ; f ; x)\right| \\
\leq \text { const } \omega_{s}\left(f ; m^{-1}\right) \log m\left[\left(\sqrt{1-x}+m^{-1}\right)^{1-2 \alpha}\right. \\
\left.+\left(\sqrt{1+x}+m^{-1}\right)^{1-2 \beta}\right], \\
\quad|x| \leq 1, \alpha, \beta \geq 0 .
\end{array}
\end{aligned}
$$

We omit the proof, since it is very similar to the proofs of the previous theorems.

\section{ACKNOWLEDGMENT}

The authors are grateful to the referees for their suggestions and remarks.

\section{BIBLIOGRAPHY}

1. A. Bellen, Metodi di proiezioni estesi, Boll. Un. Ital. Suppl. 1 (1980), 239-251.

2. A A note on mean convergence of Lagrange interpolation, J. Approx. Theory 33 (1981), $85-95$.

3. G. Criscuolo and G. Mastroianni, On the convergence of the Gauss quadrature rules for the Cauchy principal value integrals, Ricerche Mat. 35 (1986), 45-60.

4. transform, J. Approx. Theory (to appear).

5. W. Gautschi, A survey of Gauss-Christoffel quadrature formulae, E. B. Christoffel: The Influence of his Work on Mathematics and the Physical Sciences (P. L. Butzer and F. Fehér, eds.), Birkhäuser, Basel, 1981, pp. 72-147.

6. Gauss-Kronrod quadrature-A survey, Numerical Methods and Approximation Theory III (G. V. Milovanović, ed.), Prosveta, Niš, 1988, pp. 39-66.

7. H. Gonska and E. Hinnemann, Punktweise Abschätzungen zur Approximation durch algebraische Polynome, Acta Math. Hungar. 46 (1985), 243-254.

8. A. Maté, P. Nevai, and V. Totik, Extensions of Szegö's theory of orthogonal polynomials. II, Constr. Approx. 3 (1987), 51-72.

9. G. Monegato, Stieltjes polynomials and related quadrature rules, SIAM Rev. 24 (1982), 137158.

10. P. Nevai, Orthogonal polynomials, Mem. Amer. Math. Soc. No. 213 (1979).

11. _ Mean convergence of Lagrange interpolation. III, Trans. Amer. Math. Soc. 282 (1984), 669-698.

12. P. Nevai, Géza Freud, orthogonal polynomials and Christoffel functions. A case study, J. Approx. Theory 48 (1986), 3-167.

Istituto per Applicazioni della Matematica, C.N.R., Via P. Castellino, 111, 80131 NAPOLI, ITALY

Istituto di Matematica, Università della Basilicata, Via N. Sauro 85, 85100 Potenza, ITALY

Istituto per le Applicazioni del Calcolo, C.N.R., Viale del Policlinico 137, 00161 ROMA, ITALY 